\title{
Prevalência de tabagismo em estudantes de faculdade de medicina
}

\section{Prevalency of smoking in medicine students}

\author{
Rafael Thiesen Magliari', Alexandre Lima Pagliusi ${ }^{2}$, Bruno Manetti \\ Previero ${ }^{2}$, Fernando Reis Menezes ${ }^{3}$, Andre Feldman ${ }^{4}$, \\ Neil Ferreira $\mathrm{Novo}^{5}$
}

\begin{abstract}
Magliari RT, Pagliusi AL, Previero BM, Menezes FR, Feldman A, Novo NF. Prevalência de tabagismo em estudantes de faculdade de medicina. Rev Med (São Paulo). 2008 out.-dez.;87(4):264-71.

RESUMO: O tabagismo é atualmente a principal causa de enfermidades evitáveis e incapacidades prematuras em âmbito mundial, sendo que a Organização Mundial de Saúde (OMS) estima cerca de 10 milhões de mortes anuais nos próximos 30 a 40 anos em virtude de complicações de saúde decorrentes do tabaco. Apesar de possuir uma função preventiva em programas antitabagistas, estudos recentes revelam que a classe médica possui porcentagem significativa de profissionais fumantes, abalando a credibilidade da mensagem de que fumar é lesivo à saúde. Devido a diversos fatores, dentre eles as dificuldades acadêmicas impostas pelo treinamento médico, parcela significativa de médicos começa a fumar durante a faculdade. O trabalho atual tem como meta analisar a prevalência de tabagismo entre 241 acadêmicos de medicina, através de questionário validado. Verificou-se uma incidência média de $17,8 \%$ de fumantes cuja idade média foi de 21,6 anos e tempo médio de consumo de tabaco de 5,9 anos. Os autores do trabalho concluem a importância de se conhecer os dados de tabagismo dentre os estudantes de medicina consiste em reforçar a necessidade de campanhas de cessação do fumo em todas as classes da sociedade, inclusive e, principalmente, dentre os futuros profissionais médicos que serão os principais agentes na prevenção do tabagismo.
\end{abstract}

DESCRITORES: Tabaco. Nicotina. Tabagismo/epidemiologia. Estudantes de medicina.

1. Acadêmicos do 5ำ ano do Curso de Medicina da Faculdade de Medicina da Universidade de Santo Amaro.

2. Acadêmico do 6을 ano do Curso de Medicina da Faculdade de Medicina da Universidade de Santo Amaro.

3. Médico Residente de Clínica Médica da Pontifícia Universidade Católica de São Paulo.

4. Plantonista da Clínica Médica da Faculdade de Medicina da Universidade de Santo Amaro.

5. Professor titular da cadeira de Saúde Pública da Faculdade de Medicina da Universidade de Santo Amaro.

Endereço para correspondência: Rafael Thiesen Magliari. Rua Dos Democratas, 799 - Apt. 41. CEP: 04305-000 - Vila Monte Alegre, São Paulo, SP. 


\section{INTRODUÇÃO}

O tabagismo é, hoje, a principal causa de enfermidades evitáveis e incapacidades prematuras, com tendências de consumo alarmantes a despeito de todo conhecimento científico acumulado. Segundo a Organização Mundial de Saúde (OMS), para os próximos 30 a 40 anos, a epidemia tabágica será responsável por 10 milhões de mortes por ano, sendo que $70 \%$ dessas mortes ocorrerão nos países em desenvolvimento. Anualmente, cerca de cinco milhões de pessoas morrem em todo o mundo devido ao uso do tabaco'1.

Apesar de todo o conhecimento científico acumulado sobre os riscos do tabaco, as tendências no seu consumo ainda são alarmantes. No início da década de 90, cerca de 1,1 bilhão de indivíduos usavam tabaco no mundo. Em 1998, esse número já atingia a cifra de 1,25 bilhão de pessoas. Estimase que, em 2030, cerca de 1,6 bilhões de pessoas estejam em uso de tabaco no mundo².

Dentre crianças e adolescentes, as tendências de consumo desta substância é ainda mais alarmante. Segundo o Banco Mundial, quase 100.000 jovens começam a fumar a cada dia no mundo inteiro destes, mais de 80.000 são jovens de países em desenvolvimento. A idade média de iniciação no tabagismo é 15 anos, o que fez com que a OMS passasse a considerar o tabagismo uma doença pediátrica $^{3,4}$.

O consumo do tabaco é um hábito bastante freqüente na sociedade brasileira embora, mediante levantamentos pontuais, estimava-se que, em 1988, $41 \%$ dos homens adultos e $37 \%$ das mulheres adultas brasileiras eram tabagistas. Esse hábito é importante fator de risco para o desenvolvimento de inúmeras doenças, tais como: neoplasias (pulmão, laringe, esôfago, boca, bexiga, pâncreas, rim, estômago e colo uterino), doença aterosclerótica (doença coronária, doença vascular periférica, acidente vascular encefálico), doença pulmonar obstrutiva crônica e úlcera péptica ${ }^{5}$.

A classe médica tem papel muito importante nos programas antitabagismo, devendo ser mobilizada para uma atuação preventiva, com ações educativas junto à população, de modo a atuar na diminuição do número de pessoas que se iniciam no tabagismo. Entretanto, em trabalho epidemiológico feito por Mirra e cols. sobre a incidência de tabagismo na classe médica, verificou-se que dos 11.909 médicos que participaram espontaneamente do estudo 759 (6,4\%) dos profissionais da saúde são fumantes regulares e $11.150(93,6 \%)$ não são fumantes ${ }^{6}$. Dos profissionais não fumantes, 4.085 médicos (34,3\%) são exfumantes. Os maiores índices de não fumantes estão entre os associados de sociedades de especialidades que possuem programas efetivos de controle do tabagismo (pneumologia, cancerologia, cardiologia e otorrinolaringologia). O médico que fuma destrói toda a credibilidade da mensagem de que fumar é lesivo à saúde e apressa a morte, desacreditando a luta antitabágica 6 .

Segundo Menezes et al. ${ }^{7}$, em estudos realizados com estudantes de Medicina, a maioria deles mostra reduções significativas do hábito de fumar de 1980 a 1990, mas, na última década, essas reduções têm sido menos visíveis. Um trabalho realizado na Croácia não encontrou diferença significante na taxa de tabagismo em estudantes de Medicina em 1989 (31\%) e $2000(29 \%)^{8}$. Da mesma maneira, um estudo feito na Eslováquia não detectou declínio no percentual de tabagismo em estudantes de Medicina entre 1995 e $1999^{9}$.

A literatura prova que existe uma ampla variação na taxa de tabagismo dentre os estudantes de diferentes faculdades de medicina ao redor do mundo. Um estudo americano mostrou que apenas $3,3 \%$ dos estudantes de Medicina fumavam ${ }^{10}$. Em contrapartida, na Grécia 33,2\% dos estudantes do sexo masculino e $28,4 \%$ das estudantes fumavam ${ }^{11}$. Além destes, na Alemanha, a prevalência do hábito tabágico entre estudantes de Medicina foi de 23,7\% e associou-se ao tabagismo materno, mas não ao paterno $^{12}$.

Vários estudos têm demonstrado um aumento na prevalência de tabagismo ocorrida no decorrer da faculdade. Vale a pena considerar que 80 a $90 \%$ dos tabagistas atuais iniciaram o consumo de tabaco ao redor dos 18 anos, idade média compatível com o egresso nas faculdades de medicina. Dados nacionais indicam que a prevalência atual de tabagismo nos estudantes de Medicina varia de 10\% a $20 \%$. O tabagismo entre estudantes de Medicina brasileiros parece associar-se diretamente com o ano do curso, o que está de acordo com a maioria dos estudos internacionais ${ }^{7}$.

Atualmente, não resta dúvida sobre os danos provocados pelo tabagismo. Na verdade, se o tabaco tivesse sido descoberto recentemente, sua produção e venda seriam ilegais, como ocorre com outras drogas. As evidências científicas, hoje, apontam o tabagismo como uma doença gerada pela dependência da nicotina que obriga seus consumidores a se exporem a mais de 4.700 substâncias tóxicas e, desta forma, propiciam o desenvolvimento doenças graves, limitantes e fatais, como câncer e doenças cardiovasculares ${ }^{13}$. 
A fumaça do cigarro é composta por substâncias químicas voláteis $(92 \%)$ e material particulado $(8 \%)$ resultantes da combustão do tabaco. A nicotina, uma amina terciária volátil, é o componente ativo mais importante do tabaco. Cada cigarro contém 7-9 mg de nicotina, dos quais pouco mais de $1 \mathrm{mg}$ é absorvido pelo fumante ${ }^{13}$.

O tabagismo está relacionado a $30 \%$ das mortes por câncer. É fator de risco para desenvolver carcinomas do aparelho respiratório, esôfago, estômago, pâncreas, cérvice uterina, rim e bexiga. A nicotina induz tolerância e dependência pela ação nas vias dopaminérgicas centrais, levando às sensações de prazer e recompensa mediadas pelo sistema límbico. É estimulante do sistema nervoso central (SNC), aumenta o estado de alerta e reduz o apetite. A diminuição de $50 \%$ no consumo da nicotina pode desencadear sintomas de abstinência nos indivíduos dependentes: ansiedade, irritabilidade, distúrbios do sono, aumento do apetite, alterações cognitivas e fissura pelo cigarro ${ }^{14}$.

Levantamentos sistemáticos realizados pelo Centro Brasileiro de Informações sobre Drogas (CEBRID) referentes ao uso de drogas por estudantes de $1^{\circ}$ e $2^{\circ}$ graus confirmam a tendência mundial que aponta em direção a iniciação cada vez mais precoce e de forma mais pesada do uso de drogas. O levantamento sobre uso de drogas entre jovens estudantes de Salvador, realizado pelo CEBRID, revelou que $30,5 \%$ dos jovens fizeram uso do tabaco pelo menos uma vez na vida, e 5,2\% fazem uso freqüente de cigarro. Dentre os usuários que informaram o sexo, 33,5\% eram homens e 30,2\% mulheres. A maior parte dos fumantes $(42,3 \%)$ estava na faixa etária entre 16 e 18 anos, seguidos daqueles $(36,9 \%)$ com mais de 18 anos $^{15}$.

São estimadas mais de 200.000 mortes/ano decorrentes do tabagismo no Brasil ${ }^{16}$. De forma diferente ao que acontece com a maioria dos problemas de saúde pública, o tabagismo conta com um importante fator causal ligado a um negócio: o comércio de produtos de tabaco. Em virtude do impacto social e econômico do tabagismo na sociedade atual, bem como ao sistema público de saúde, e a crescente preocupação em se tentar abolir ou reduzir o consumo de tabaco os autores propuseram a realização deste estudo.

\section{OBJETIVO}

Analisar a prevalência de tabagismo entre os acadêmicos de medicina de acordo com o sexo, bem como o perfil do hábito tabágico entre os acadêmicos de medicina tabagistas da Faculdade de Medicina de Santo Amaro.

\section{MATERIAL E MÉTODO}

Através da aplicação de questionário (Anexo 1), realizou-se a coleta de dados com estudantes de medicina do $1^{\circ}$ ao $5^{\circ}$ ano da Faculdade de Medicina da Universidade de Santo Amaro em janeiro de 2008. Em um universo de 302 estudantes, $241(79,8 \%)$ participaram da pesquisa. Os alunos do $6^{\circ}$ ano não foram incluídos no estudo devido a pouca adesão, 6 em um total de 75 estudantes. A formulação deste questionário de tabagismo baseou-se no questionário de tolerância de Fagerstrom ${ }^{17}$ amplamente utilizado na literatura sendo este constituído por cinco questões, que visam identificar o comportamento dos fumantes e seu grau de dependência. O questionário, uma vez obtida a aceitação do estudante para aplicação, foi preenchido pelos internos participantes deste estudo.

Definiu-se como fumante o estudante que fumava mais de um cigarro por dia há mais de um mês. Os indivíduos fumantes responderam a todas as questões e os não fumantes apenas deixaram de responder àquelas relacionadas ao hábito de fumar.

A análise estatística foi realizada através do teste do qui quadrado com o objetivo de estudar as associações entre o hábito do tabagismo com as demais variáveis estudadas. O mesmo teste foi aplicado para comparar os gêneros feminino e masculino em relação ao desejo e a tentativa de parar de fumar, tempo de tabagismo e número de cigarros por dia.

\section{RESULTADOS}

Dos 241 estudantes, sendo estes 164 do sexo feminino $(68,8 \%)$ e 77 do sexo masculino $(31,2 \%)$, encontrou-se uma incidência média de $17,8 \%$ de fumantes cuja idade média foi de 21,6 anos e tempo médio de consumo de tabaco de 5,9 anos. Observou-se que a incidência de tabagismo entre os indivíduos do sexo feminino é de 15,8\% enquanto que, no sexo masculino, este valor é de 22\% (Gráfico 1, Tabela 1).

Dentre os fumantes, encontrou-se que $60 \%$ eram do sexo feminino e $40 \%$ do sexo masculino. Observou-se que não existe relação entre o hábito de fumar e a prática ou não regular de esportes (Tabela 2). 
GRÁFICO 1. Prevalência de tabagismo entre os sexos

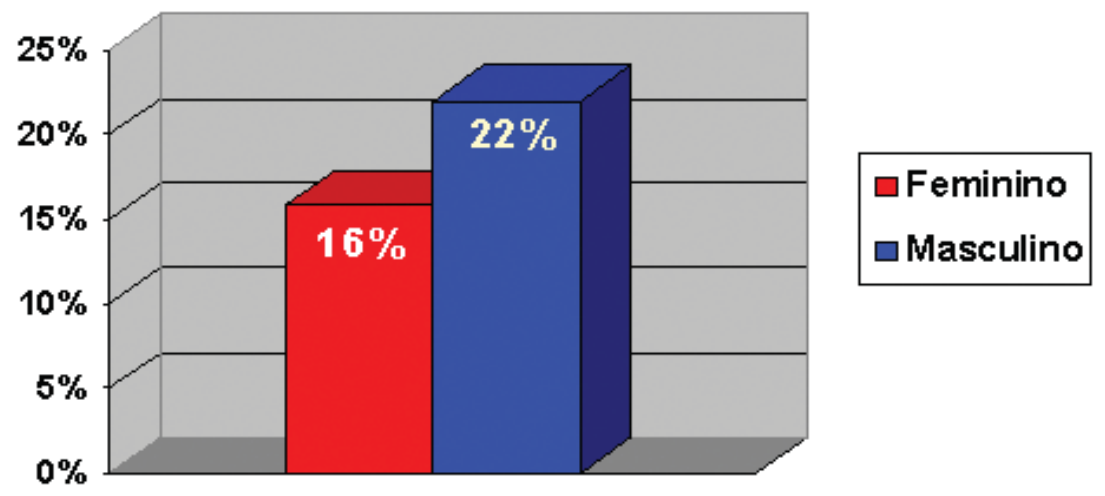

TABELA 1. Prevalência do tabagismo por sexo

\begin{tabular}{lcccc}
\hline & Fuma & Não fuma & Total & Porcentagem (\%) \\
\hline Masculino & 17 & 60 & 77 & 22,1 \\
Feminino & 26 & 138 & 164 & 15,8 \\
Total & 43 & 198 & 241 & 17,8 \\
\hline $\mathrm{X}^{2}=1,38(\mathrm{~ns})$ & & & &
\end{tabular}

TABELA 2. Relação entre fumantes que praticam esportes

\begin{tabular}{lccccccc}
\hline \multicolumn{7}{c}{ Mulher } & \multicolumn{7}{c}{ Homem } \\
\hline Esporte & Fuma & Não fuma & Total(\%) & Fuma & Não fuma & Total(\%) & $X^{2}$ \\
Pratica & 10 & 50 & $60(16,4)$ & 11 & 36 & $47(23,4)$ & $0,83(\mathrm{~ns})$ \\
\hline Não Pratica & 16 & 88 & $104(15)$ & 6 & 24 & $30(20,0)$ & $0,50(\mathrm{~ns})$ \\
\hline Total & 26 & 138 & $164(15,8)$ & 17 & 60 & $77(21,1)$ & \\
\hline
\end{tabular}

$\mathrm{X}^{2}=0,03(\mathrm{NS}) ; \mathrm{X}^{2}=0,41(\mathrm{NS})$

Os autores puderam observar que mais de $50 \%$ dos estudantes tabagistas gostariam de parar

TABELA 3. Desejo de parar de fumar em relação ao sexo

\begin{tabular}{lcccc}
\hline Sexo & Sim & Não & Total & Porcentagem (\%) \\
\hline Homem & 10 & 7 & 17 & 58,8 \\
Mulher & 15 & 11 & 26 & 57 \\
Total & 25 & 18 & 43 & 58,1 \\
\hline $\mathrm{X}^{2}=0,01(\mathrm{NS})$ & & & &
\end{tabular}

definitivamente e que cerca de $46,5 \%$ dos estudantes já tentaram parar de fumar sem sucesso (Tabelas 3 e 4).

TABELA 4. Tentativa de parar de fumar em relação ao sexo

\begin{tabular}{lcccc}
\hline Sexo & Sim & Não & Total & Porcentagem (\%) \\
\hline Homem & 9 & 8 & 17 & 52,9 \\
Mulher & 11 & 15 & 26 & 42,3 \\
Total & 20 & 23 & 43 & 46,5 \\
\hline $\mathrm{X}^{2}=0,47(\mathrm{~ns})$ & & & &
\end{tabular}


Quando se analisou a prevalência de tabagismo por ano de graduação, observou-se uma maior prevalência de tabagismo no quinto ano da amostra analisada com $32 \%$ dos tabagistas. A média de tabagismo dos outros anos variou entre $9,6 \%$ a $16 \%$ (Tabela 5). Observou-se que, quanto ao tempo de duração do tabagismo, mais de $70 \%$ dos tabagistas acadêmicos já o faziam por mais de 4 anos de duração. Apenas $28 \%$ dos acadêmicos são tabagistas por menos de 4 anos (Tabela 6).

TABELA 5. Tabagismo por ano da graduação

\begin{tabular}{lcccc}
\hline Ano & Fuma & Não fuma & Total & $\begin{array}{c}\text { Porcentagem } \\
\text { (\%) }\end{array}$ \\
\hline 10 & 5 & 47 & 52 & 9,6 \\
\hline 20 & 8 & 42 & 50 & 16 \\
\hline 3 & 3 & 22 & 25 & 12 \\
\hline 4ㅇ & 6 & 44 & 50 & 12 \\
\hline 5 & 21 & 43 & 64 & 32,8 \\
\hline
\end{tabular}

$\mathrm{X}^{2}=14,05$ (Significativo para o $5^{\circ}$ ano)

TABELA 6. Tempo de duração do hábito de fumar

\begin{tabular}{ccc}
\hline Tempo & Total & Porcentagem (\%) \\
\hline 2-4 anos & 12 & $28 \%$ \\
\hline 4-7 anos & 14 & $33 \%$ \\
\hline 7-10 anos & 9 & $21 \%$ \\
\hline 10-14 anos & 8 & $18 \%$ \\
\hline
\end{tabular}

O número de cigarros consumidos ao dia tem relação direta com o surgimento de diversas doenças. $\mathrm{Na}$ amostra analisada, constatou-se que o consumo de até um maço de cigarros é realizado por cerca de 91\% dos acadêmicos (Tabela 7, Gráfico 2).

TABELA 7. Número de cigarros por dia em relação ao sexo

\begin{tabular}{lccc}
\hline Cigarros & Homem & Mulher & Total (\%) \\
\hline $1-10$ & 9 & 17 & $26(62 \%)$ \\
$11-20$ & 4 & 8 & $12(29 \%)$ \\
\hline $21-30$ & 3 & 0 & $3(7 \%)$ \\
\hline $31-40$ & 1 & 0 & $1(2 \%)$ \\
\hline
\end{tabular}

$\mathrm{X}^{2}=$ Não analisável

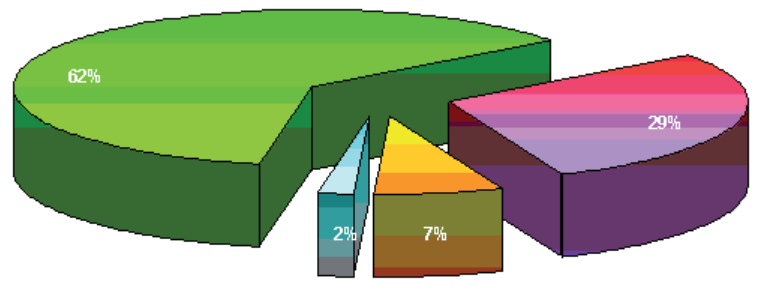

$\square 1$ a $10 \square 11$ a $20 \square 21$ a $30 \square 31$ a 40

GRÁFICO 2 - Prevalência de fumantes de acordo com a carga tabágica diária

\section{DISCUSSÃO}

Dos $241(79,8 \%)$ estudantes de medicina que responderam ao questionário, encontrou-se uma idade média de 21,6 anos e uma prevalência de $17,8 \%$ de fumantes, que aproxima-se ao valor médio encontrado na literatura que gira em torno $22 \%$. Estudo realizado na Faculdade de Medicina de Sorocaba, em 1989, mostrou prevalência de $17,5 \%$ entre os estudantes daquela instituição. Outro estudo, realizado na Faculdade de Medicina da Universidade Federal de Pelotas, mostrou uma prevalência de $10,1 \%$ em levantamentos entre os anos de 1991 e $1996^{7}$.

Neste estudo pôde-se encontrar que não há relação entre a prevalência de tabagismo em indivíduos que praticam e aqueles que não praticam atividade física. Na literatura não existem dados que avaliam este tipo de evento em estudantes.

Dentre os fumantes, encontrou-se que $39,5 \%$ eram do sexo masculino e $60,5 \%$ do sexo feminino. Esta falsa aparência de maior consumo de tabaco em acadêmicas se dá devido ao ainda maior número de estudantes do sexo feminino nas Universidades de Medicina, perfil este que vêm se mantendo nos últimos anos. No presente estudo, pela maioria da amostra ser de estudantes do sexo feminino, a prevalência de tabagismo neste gênero foi maior do que em relação ao sexo masculino. Sabe-se, no entanto, que a prevalência de tabagismo é maior no sexo masculino (60\%) em relação ao sexo feminino (40\%). Porém, estudos mais recentes, mostram crescimento do tabagismo por entre indivíduos do sexo feminino e tendência à redução do tabagismo entre homens. Esta tendência levará a profundas mudanças na distribuição do tabaco entre os sexos nos próximos anos ${ }^{5,6}$.

Os autores puderam observar que $58 \%$ dos estudantes do sexo masculino e $57 \%$ do sexo feminino gostariam de parar definitivamente com o consumo de tabaco e que cerca de $46,5 \%$ dos estudantes já tentaram parar de fumar sem sucesso. 
Estudos mostram que os indivíduos que efetivamente conseguem interromper o hábito do tabagismo já passaram por 3 a 4 tentativas prévias sem sucesso. Desta forma, é comum que haja diversas tentativas previamente ao sucesso da interrupção definitiva do hábito tabágico ${ }^{18}$. Em comparação à literatura, um estudo espanhol realizado com estudantes do sexto ano das faculdades de medicina demonstrou que $76,3 \%$ dos fumantes declararam que gostariam parar de fumar ${ }^{19}$.

Com relação ao ano de graduação dos estudantes, os autores observaram que os estudantes do quinto ano obtiveram a maior prevalência de tabagismo $(32,8 \%)$ em comparação aos estudantes do primeiro ano $(9,6 \%)$. Este fato talvez possa deverse em parte à influência sofrida pelos amigos e, por uma considerável parcela dos estudantes mais velhos morarem fora de casa. Os estudantes situados nos anos intermediários obtiveram semelhante prevalência (segundo ano: $16 \%$, terceiro ano: $12 \%$ e quarto ano: $12 \%)$, porém deve-se atentar ao fato que o número de entrevistados do terceiro ano foi praticamente a metade dos outros anos. O mesmo estudo espanhol de Mas e colaboradores demonstrou que $32,54 \%$ dos fumantes do sexto ano de medicina iniciaram o hábito do tabagismo após ingressarem na faculdade de medicina e que a prevalência de tabagismo aumenta com o passar dos anos de faculdade ${ }^{18}$.

Os autores observaram que mais de $70 \%$ dos estudantes fumam há pelo menos 4 anos, dados estes equiparáveis aos encontrados na literatura que mostram que $80 \%$ dos estudantes iniciam o hábito tabágico por volta dos 15 anos $^{3}$.

A maioria dos estudos mostraram que o uso de mais de dois cigarros ao dia já é prejudicial ao organismo e pode levar à dependência ${ }^{14}$. Neste estudo mostrou-se que mais de $90 \%$ dos estudantes fumam até 1 maço de cigarro (20 cigarros) ao dia que é considerada uma elevada carga tabágica sendo extremamente prejudicial à saúde.

\section{CONCLUSÃO}

Os autores concluem que a prevalência de tabagismo encontrada na Faculdade de Medicina de Santo Amaro é semelhante àquela observada em outras faculdades de medicina estando esta, abaixo da incidência de tabagismo na população geral que gira em torno de $24 \% .{ }^{13}$ No mesmo sentido, diferentemente da população em geral, os médicos têm uma função importante na prevenção e erradicação do tabagismo na comunidade. Por isso, a prevalência de tabagismo em estudantes de medicina e médicos deveria ser ainda menor.

$\mathrm{Na}$ população analisada, a prevalência de tabagismo é maior em mulheres. Este dado reforça as recentes tendências observadas de crescimento do tabagismo no sexo feminino também entre as estudantes de medicina.

Foi observado que o número de tabagistas é maior nos estudantes nos anos finais da graduação comparado àqueles que estão nos primeiros anos do curso. Ao mesmo tempo em que a prevalência de tabagismo parece aumentar ao longo da graduação, a porcentagem de tabagistas querendo interromper este hábito supera a metade da população de tabagistas. Infelizmente, constatou-se que a carga tabágica dos acadêmicos de medicina é alta, girando em torno de 1 maço de cigarros ao dia em cerca de $90 \%$ dos tabagistas.

Os autores finalizam reforçando a importância de se conhecer os dados de tabagismo dentre os estudantes de medicina consiste em reforçar a necessidade de campanhas de cessação do tabagismo em todas as classes da sociedade, inclusive e, principalmente, dentre os futuros profissionais médicos que serão os principais agentes na promoção de interrupção do tabagismo na população geral em um futuro próximo.

Magliari RT, Pagliusi AL, Previero BM, Menezes FR, Feldman A, Novo NF. Prevalency of smoking in medicine students. Rev Med (São Paulo). 2008 out.-dez.;87(4):264-71.

ABSTRACT: This is a epidemiological paper concerning the use of cigarettes by medicine students and also a literature review based on several different articles. According to the World Health Organization (WHO), smoking is the main cause of preventable diseases in the planet and is directly responsible for about 8 millions deaths a year. Such numbers aren't a big surpise for any educated reader, but what is interesting is that despite this, the number of smokers are progressively growing. The medical class, which are the people responsible for informing and warning the population have a great percentage of smokers. The present paper illustrate this fact, through a questionnaire answered by 241 students with a percentage of $17.8 \%$ smokers. This fact may be related to the stress involved in the medical course, associated with lack of time in their private lives. The author concludes that having reliable smoking data about the medicine students is of vital importance, demonstrating the need of emphases in prevention campaigns in all society classes, especially among health students, who will be the main agents in promoting health in the future.

KEY WORDS: Tobacco. Nicotine. Smoking/epidemiology. Students, medical. 
Anexo 1. Questionário aplicado aos estudantes de medicina

\section{PESQUISA SOBRE TABAGISMO}
1) Idade:
2) Sexo: $M($ ) $F($ )
3) Estado Civil: ( ) Solteiro(a) ( ) Casado(a) ( ) Viúvo(a) ( ) Separado(a)
4) Escolaridade:
5) Seus pais fumam? ( ) S ( ) N
6) Se não fumam, foram fumantes? ( ) S ( ) N
7) Você fuma? ( ) S ( ) N
8) Há quanto tempo?
9) Quantos cigarros por dia atualmente?
10) Quanto tempo depois de acordar você fuma seu $1^{\circ}$ cigarro?
11) Você fuma mais freqüentemente pela manhã? ( ) $S \quad($ ) N
12) Vc acha difícil não fumar em lugares proibidos como igrejas, cinemas, ônibus, biblioteca, etc?
( ) S ( ) N
13) Você fuma mais quando está sozinho? ( ) S ( ) N
14) Gostaria de parar de fumar? ( ) S ( ) N
15) Já tentou parar de fumar? ( ) S ( ) N
16) Se sim, pq não conseguiu?
17) Pratica esporte (mais de $3 x$ por semana por pelo menos 30 minutos)? ( ) S ( ) N

\section{REFERÊNCIAS}

1. Lemos Junior AV, Silva M, Lessa PP, Costa RC, Colosio T. Estratégias antitabágicas. Rev Med UCPEL. 2004;2(1):32-37.

2. World Health Organization (WHO). Confronting the tobacco epidemic in an era of trade liberalization. Geneva; 2001. WHO/NMH/TFI/01.4.

3. Malcon MC, Menezes AMB, Chatkin M. Prevalence and risk factors for smoking among adolescents. Rev. Saúde Pública. 2003;37(1):1-7.

4. Banco Mundial. A epidemia do tabagismo: os governos e os aspectos econômicos do controle do Tabaco. The World Bank; 1999.

5. Lolio CA, Souza JMP, Santo AH, Buchalla CM. Prevalence of smoking in a city of southeasthern Brazil. Rev. Saúde Pública. 1993;27(4):262-5.

6. Mirra AP, Rosemberg J. Survey on prevalence of smoking among Brazilian physicians. Rev Assoc Med Bras. 1997;43(3):209-16.

7. Menezes AMB, Hallal PC, Silva F, Souza M, Paiva L, D'ávila A, et al. Tabagismo em estudantes de medicina: tendências temporais e fatores associados. J Bras Pneumol. 2004;30(3):223-8.

8. Trkulja V, Zivcec Z, Cuk M, Lackovic Z. Use ofpsychoactive substances among Zagreb
Universitymedical students: follow-up study. Croat Med J. 2003;44:50-8.

9. Baska T, Straka S, Madar R. Smoking and some lifestylechanges in medical students - Slovakia, 1995-1999. Cent Eur J Public Health. 2001;9:147-9.

10. Patkar AA, Hill K, Batra V, Vergare MJ, Leone FT. Acomparison of smoking habits among medical andnursing students. Chest. 2003;124:1415-20.

11. Mammas IN, Bertsias GK, Linardakis M, Tzanakis NE, Labadarios DN, Kafatos AG. Cigarette smoking, alcohol consumption, and serum lipids profile among medical students in Greece. Eur J Public Health. 2003;13:278-82.

12. Brenner H, Scharrer SB. Parental smoking and sociodemographic factors related to smoking among German medical students. Eur J Epidemiol. 1996;12:171-6.

13. Brasil. Ministério da Saúde. Instituto Nacional do Câncer. Por um mundo sem tabaco: mobilização da sociedade civil. Ação global para o controle do tabaco o 1o Tratado Internacional de Saúde Pública. 3a ed. Rio de Janeiro: INCA; 2004. Disponível em: http://www. inca.gov.br/tabagismo/cquadro3/acao_global.pdf

14. Balbani APS, Montovani JC. Métodos para abandono do tabagismo e tratamento da dependência da nicotina. Rev Bras Otorrinolaringol. 2005;71(6):820-7.

15. Galduróz JCF, Noto AR, Carlini EA. IV Levantamento 
sobre uso de drogas entre estudantes de $1^{\circ}$ e $2^{\circ}$ graus em 10 capitais brasileiras, 1997. São Paulo: Centro Brasileiro de Informações sobre Drogas Psicotrópicas, UNIFESP; 1997.

16. Brasil. Ministério da Saúde. Coordenação Nacional de DST/AIDS. Levantamento nacional sobre prevenção de DST/AIDS e uso indevido de drogas em escolas. Brasília; 2000.

17. Heatherton TF, Kozlowski LT, Frecker RC, Fagerström KO. The Fagerström test for nicotine dependence: a revision of the Fagerström Tolerance Questionnaire. Br J Addict. 1991;86:1119-27.

18. Cury Júnior AJ, Labbadia EM. Intervenções para o abandono do tabagismo Sociedade de Cardiologia do Estado de São Paulo. SOCESP: cardiologia. Rio de Janeiro: Atheneu; 1996. p.412-6.

19. Mas A, Nerín I, Barrueco M, Cordero J, Guillén D, Jiménez-Ruiz $C$, et al. Smoking habits among sixthyear medical students in Spain. Arch Bronconeumol 2004;40(9):403-8. 